\title{
Shift Work Disorder - Prevalence \& Burden of Illness in Healthcare Providers of India
}

\author{
Dharmik Vora ${ }^{\star}$, Sanjay Mehta and Shashank Patil
}

Emergency Medicine, Chelsea \& Westminster Hospital NHS

${ }^{*}$ Corresponding author

Dr. Dharmik Vora, Emergency Medicine, Chelsea \& Westminster Hospital NHS Foundation Trust, United Kingdom. E-mail: dharmik.vora@nhs.net

Foundation Trust, United Kingdom

Submitted: 11 June 2019; Accepted: 20 June 2019; Published: 29 June 2019

\begin{abstract}
Background: The proportion of shift workers in the society has taken a big leap in the last 2 decades or so. Thus, the epidemiological significance of shift work disorder (SWD) can no longer be ignored. Emergency Physicians \& nurses almost always work in shifts and are an important subset of the population whose health and wellbeing directly and indirectly impacts the morbidity and mortality of the rest of the population. It is thus important to understand the magnitude of the problem in this population. Several studies describe a high incidence of SWD and psychosomatic complaints in EP. The main objective of this study is to examine the prevalence of symptoms of SWD; contribution of demographics, working hours, shift work, morningness/eveningness \& sleep hygiene practices to occurrence of SWD, related health \& occupational hazards and job satisfaction in a random sample of Emergency Physicians \& nurses of India.
\end{abstract}

\begin{abstract}
Methods: A cross sectional survey of nurses and doctors working in emergency departments of at least 12 major urban hospitals across India was conducted during October 2016 - March 2017. Peer validated questionnaire with standard scales, descriptive \& objective questions was emailed only to individuals who were known to be working in Emergency departments as nurses and doctors. This convenience-sample of email addresses was obtained through personal and professional contacts of the researcher. Those who volunteered responses were included in the study. No identifying information was collected. Those who reported diagnosed sleeping disorders were excluded from the analysis. For analysis, responses were divided into 2 groups - those who reported symptoms of SWD and those who did not. With aid of a professional biostatistician, these 2 groups were then compared for unique characteristics and statistically significant variables using t test, chi square test, odds ratio and logistic regression
\end{abstract}

Results: Prevalence of symptoms indicative of SWD in a random sample of emergency medicine physicians and nurses in India ranges from 13-27\% and was significantly $(p=0.048)$ higher in those who did shift work and night shift work depending on the method of assessment. Women have 3 times higher risk than men. More than half (51\%) the number of Emergency medicine physicians and nurses in the study suffer sleep disturbances due to work timings. This group may develop SWD in the future, pending due intervention. SWD is strongly $(p<0.02)$ associated with bad sleep hygiene and excessive daytime sleepiness. The presence of symptoms of SWD also leads to poor job satisfaction. $(p<0.05)$. Majority $(60 \%)$ report preference for shifts that start later in the day.

\section{Abbreviations}

SWD: Shift Work Disorder

ICSD: International classification of sleep disorders

ED: Emergency Department

EM: Emergency medicine

EP: Emergency Physician

ESS: Epworth Sleepiness Scale

SHI: Sleep Hygiene Index

NSW: Night Shift Work

Introduction and Background

Emergency Physicians as individuals are involved in the acute care of critical patients in a time/resource/space constrained environment and one split second decision of EP can play an instrumental role in outcome of care being given. Our ED had approximately 50000 visits in the last 3 years. Many of our patients are highly valuable human resources of our community, city and country. Thus, sensibility of our decision snowballs into a big impact on the individual, community and country in terms of morbidity, mortality, time, cost, productivity and innumerable abstract factors like physician/ hospital perception, feelings of personal and community well being, and even overall general outlook of the individual. Hence it is absolutely imperative that Emergency physician be fully alert, energetic, non-groggy, and suffer least or no symptoms of avoidable physical, mental and emotional energy drain in order to minimise cognitive, behavioural, motor, concentration and communication 
lapses during administering patient care. In order to achieve the same, it is necessary to understand the stressors which affect the practice of Emergency Medicine. Residency training in any medical specialty comes with its own set of stressors. Even that needs to be taken into consideration.

A variety of stressors contribute to the potentially unhealthful aspects of EM practice, both during residency training and in practice $[1,2]$. These stressors generally relate to four aspects of emergency practice:

1. Difficult patient and professional relationships,

2. Diversity of practice elements,

3. Diminished resources,

4. Difficult decisions full of uncertainty.

\section{Residency stress relates to}

1. Time demands and work hours

2. Autonomy

3. Balance between personal and professional goals

4. Fear of personal safety and well-being.

\section{Consequence: Burned out or Impaired physician}

Burnout can be categorized along three general dimensions: emotional exhaustion (feeling emotionally drained by contact with other people), depersonalization (negative feelings toward patients receiving care), and reduced personal accomplishment (the tendency to reflect negatively on your own work) $[3,4]$. Burnout is of special relevance to physicians because it undermines the integrity of the physician-patient relationship, which is the foundation of medical practice and essential to effective service. People in advanced phases of burnout are likely to experience decreased productivity, less satisfaction with work, higher job turnover, lower self-esteem, more physical symptoms, more troubled family relationships, and a variety of affective changes, such as hostility, anxiety, depersonalization, cynicism, and depression; thus encompassing overall wellbeing and existence itself. Symptoms of burnout are thought to be potential precursors of more severe manifestations of impairment, including alcoholism, drug abuse, and suicide [5].

The Federation of State Medical Boards, USA, defines an impaired physician as one who is unable to practice medicine with reasonable skill and safety because of a mental illness; a physical illness or condition that adversely affects cognitive, motor, or perceptive skills; or substance abuse. Impairment in a physician colleague often is difficult to detect. The recognition of a pattern of events, rather than a single precipitating incident, may be key to making the diagnosis.

Difficulties that arise in the workplace often are preceded by a history of family difficulties, including frequent arguments, periods of separation, extramarital affairs, and divorce. Frequent job changes ("geographic cure") and unexplained time intervals between periods of professional employment also may be signs of impairment. A high value usually is placed on maintaining the source of income, and erosion of hospital duties is one of the last things that may be affected.

Patient care responsibilities become neglected, and the impaired physician may exhibit poor medical judgment. The problem may develop during a long period because strong elements of denial are usual among family members and associates as well as in the impaired physician [6].
The very qualities that characterise medically successful physiciansperfectionism, the drive to succeed, willingness to work long and irregular hours, and ideals of individual service and sacrifice-also may predispose them to neglect their own physical and emotional needs. The formulation of any comprehensive well-ness strategy is based on the concept of lifestyle balance and involves the integration of professional goals and responsibilities with needs for self-care and development $[7,8]$. Cultivation of life-style balance involves four basic elements, summarized below.

1. Promote wellness in the professional environment.

A. General measures

B. Strategies for shift work

C. Strategies for managing difficult and violent patients.

D. Professional support groups.

2. Cultivate close family and social relationships.

3. Develop and maintain physical fitness.

4. Cultivate methods of relaxation and renewal.

Why Shift Work?

It is beyond the scope of professional intervention of the medical fraternity to modify above $[2,3,4]$. However, promoting wellness in work environment by assessing time and work demands and implementing strategies for shift work is easily possible and it's high time we stop ignoring physician health and wellbeing.

The first step in this process is to understand the magnitude of the problem in the target population.

\section{Review of literature}

Literature search was performed on PubMed using term - Shift work disorder and sought to obtain studies relevant to epidemiology of Shift work disorder. After modifying search with various terms like epidemiology, incidence, prevalence, emergency medicine, physicians, nurses, India, found 454 articles describing shift work disorder and associations like 'Prevalence, predictors, depression, anxiety, attention, coronary heart disease, hypertension, excessive sleepiness, insomnia, age, chronic disease, red cell distribution width, Parkinson's disease, multiple sclerosis, pharmacological interventions, physiological functions, periodontal health, vascular events, asthma, GERD, dyspepsia, cancer, CKD, metabolic disease; neuroendocrine, immune and oxidative stress, menstrual characteristics and an endless exhaustive list of associations have even studied and described.

\section{Search term: Shift Work Disorder}

Filters: last 5 years, adults 19+, humans, english language, all types of studies included.

Additional terms: Emergency medicine, doctors, nurses, physicians, India

After extensive search, I found a comprehensive review on shift work disorder in Supplement to the Journal of Family Practice VOL.59 NO 1/ JANUARY 2010 that describes socioeconomic burden, characterization and pathophysiology, recognition and management of shift work disorder.

Shift work disorder is defined in the third edition of the International Classification of Sleep Disorders (ICSD-3) as follows [9]. 
1. There is a report of insomnia and/or excessive sleepiness, accompanied by a reduction of total sleep time, which is associated with a recurring work schedule that overlaps with the usual time for sleep.

2. The symptoms have been present and associated with the shift work schedule for at least three months.

3. The symptoms cause clinically significant distress or impairment in mental, physical, social, occupational, education, or other important areas of functioning.

4. Sleep log and actigraphy monitoring (whenever possible and preferably with concurrent measurement of light exposure) for at least 14 days (work and free days) demonstrate a disturbed sleep and wake pattern.

5. The sleep and/or wake disturbance are not better explained by another current sleep disorder, medical or neurologic disorder, mental disorder, medication use, poor sleep hygiene, or substance use disorder.

The irregularity of scheduled hours is a major workplace issue for emergency physicians. Shift changes often are cited as a major cause of dissatisfaction within the specialty $[10,11]$. Shift work is associated with a variety of adverse effects on physical and emotional health [12-14]. These include an increased incidence of peptic ulcer disease, depression, mood swings, drug and alcohol abuse, altered immune response, chronic hypertension, cardiovascular mortality, infertility in women, divorce, work-related accidents and errors, and compromised learning ability when

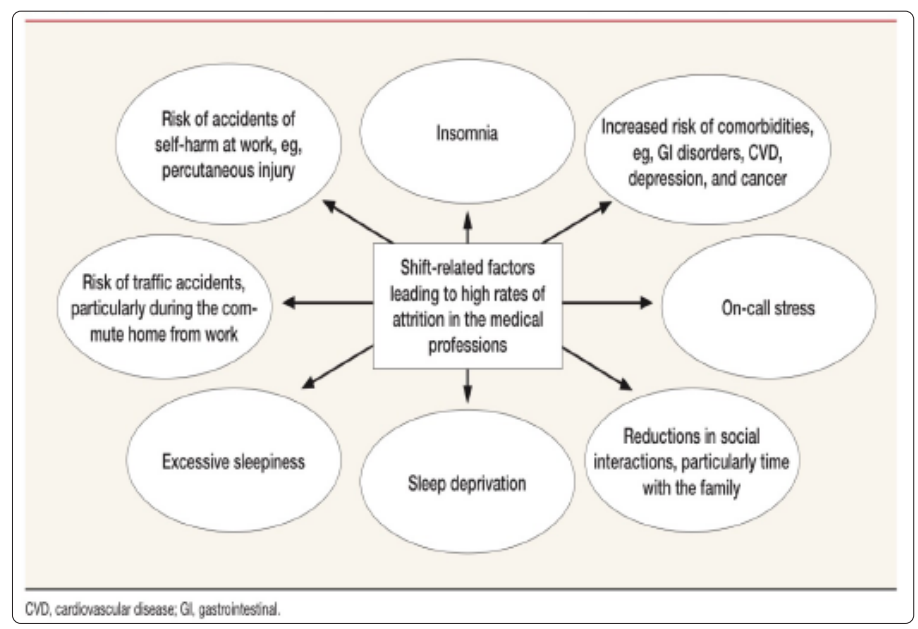

Figure 2: Shift-related factors likely to affect attrition in the emergency medical professions

Fatigued [15]. In addition, shift workers are more likely to sustain work-related accidents (e.g., percutaneous injury) [16]. Approximately $25 \%$ of persons in the North American population are shift workers, and research has shown that an estimated $20 \%$ of people cannot tolerate shift work [17-19].

Sleep deprivation makes it difficult to maintain stable performance over time, as measured by response latency, response variability, speed, accuracy, handeye coordination, decision-making, and memory [20,21].

Lacunae in literature

Limited studies were found involving Physicians and almost none on Indian population. Even though adequate research has been done on SWD, there is little data on the prevalence and impact - burden of illness of SWD in emergency medicine providers.

\section{Research question}

What is the burden of illness of SWD in emergency medicine physicians and nurses in terms of prevalence of symptoms of SWD, personal health, occupational errors or accidents, interpersonal relationships and job satisfaction etc.?

Aims and Objectives

Primary: What is the prevalence of Shift Work Disorder in Emergency physicians and nurses and what are its contributing factors and consequences?

\section{Secondary:}

1. To promote awareness /understanding of shift work sleep disorder, sleep hygiene and adverse effects of chronic sleep deprivation.

2. To discover the proportion of people with early phase cycle (morning-ness) vs delayed phase sleep cycle (Evening-ness).

3. To make recommendations for optimizing shift work strategies based on individual preference for morning-ness vs eveningness.

\section{Material and methods}

A. Study area: Emergency medicine, India.

B. Study population: Physicians and nurses who are working in shifts in emergency departments, not limited by geography. Convenience sampling.

C. Sample size: Based on the literature, it was found that the prevalence of shift work disorder was $10 \%$ in random population. For our sample size considering prevalence of shift work disorder was to be $10 \%$ in our study population [22]. With a $5 \%$ allowable variation, the sample size at $95 \%$ confidence level; require to en-roll 140 positive participants in this study.

If $\mathrm{N}$ is the sample size for infinite population, $\mathrm{Z} 2=1.96, \mathrm{p}=0.10$, $(1-p)=0.90$ and $e 2=0.05=\sim 139$ rounded off to 140 positive participants.

D. Study design: It is a cross-sectional, epidemiological, prospective, observational study.

\section{Inclusion Criteria}

1. Age group: 18 and above

2. Gender: Male and female volunteers will be included

3. Volunteers who agree to fill the questionnaire form.

4. Volunteer should be a physician or a nurse working in the emergency department for at least 6 months.

Exclusion Criteria: Individuals with known medical/psychiatric conditions that affect natural sleep (eg. OSA, narcolepsy, etc.)

E. Study Intervention: Self designed, peer validated questionnaire was circulated via email. Email addresses were obtained through personal and professional contacts, online support groups, social media groups, online emergency medicine societies. Data was recorded in google forms. No identifying information was collected. Single entry per participant was ensured by enabling google account sign in. Data obtained as such were analysed with the help of a professional bio statistical expert using 
methods as explained in the following section of statistical methods.

F. Validation of Questionnaire: Questionnaire was designed to include sociodemographic data, ICSD defined diagnostic criteria of SWD, validated objective scale to assess daytime sleepiness - Epworth Sleepiness Scale, personal sleeping habits- Sleep Hygiene Index, personal habits like caffeine, alcohol consumption, etc., medical conditions, work related errors, accidents, job satisfaction and few other variables. Multiple editions of questionnaire were circulated among peers and guides to assess feasibility, ease of understanding and filling the form as well as relevance of questions included. Final questionnaire is prepared after collaboration with Emergency medicine experts namely Dr. Sanjay Mehta, Dr. Sonal Batra, Dr. Shashank Patil and Dr. Aisha Liferidge.

G. Study Duration: Responses obtained between October, 2016 - March, 2017 were included in the analyses.

\section{H. Data Collection Form: As per Annexure enclosed.}

\section{Statistical method and tools}

\section{Procedure and participants}

A sample of 216 physicians and nurses working in emergency departments across India were included in the study. The questionnaire was circulated via email. Email addresses were obtained from personal and professional contacts, professional Emergency medicine societies and George Washington university database of MEM residents and consultants in emergency medicine. The responses were recorded anonymously in google forms, which were then analyzed by a professional bio statistician using methods described in the respective section.

Following variables were compared between people who reported shift work disorder and those who did not - age, gender, BMI, designation, years of experience, duration of shift work, morningness vs eveningness, weekly working hours, average number of night shifts per week in the last 6 months, job satisfaction, substance use, medications, sleeping aids, health and occupational problems, meant total ESS scores and SHI scores.

\section{Instruments}

Shift work disorder

The present study employed questions previously developed and used specifically to assess/diagnose SWD in epidemiological studies: Participants were asked to select which one or more out of the below applies to them in the last 3 months

1. Insomnia/excessive sleepiness due to reduced sleep time, because of work timings.

2. Above symptoms cause significant distress in mental, physical, social, occupational, education or other important areas of functioning.

3. Above symptoms are present for more than 3 oaths due to shift work

4. My sleep disturbance from another cause, not related to work e.g. Medical, social, psychiatric, etc.

5. NONE - I have no sleep related trouble

These questions adhere to SWD symptoms/criteria listed in the ICSD-3. Respondents had to check 1st two boxes in order to fulfill the criteria for SWD caseness. Participants who reported sleep disturbance from other causes were included in the other sleep disorders group. Participants who reported "NONE" were included in the healthy group. As we did not perform clinical interviews, actigraphic measurements or administered sleep diaries, we did not diagnose the participants, but rather confirmed symptoms indicative of SWD.

\section{Epworth Sleepiness Scale (ESS)}

Dr Johns first developed the ESS for adults in 1990 and subsequently modified it slightly in 1997. He developed it so he could assess the 'daytime sleepiness' of the patients in his own private practice of Sleep Medicine. He named the questionnaire after Epworth Hospital in Melbourne, where he established the Epworth Sleep Centre in 1988.

The ESS is a self-administered questionnaire with 8 questions. Respondents are asked to rate, on a 4-point scale (0-3), their usual chances of dozing off or falling asleep while engaged in eight different activities. Most people engage in those activities at least occasionally, although not necessarily every day. The ESS score (the sum of 8 item scores, 0-3) can range from 0 to 24. The higher the ESS score, the higher that person's average sleep propensity in daily life (ASP), or their 'daytime sleepiness'. The questionnaire takes no more than 2 or 3 minutes to answer. It is available in many different languages.

\section{What the ESS Measures}

The ESS asks the respondent to rate on a 4-point scale (0-3) their usual chances of having dozed off or fallen asleep while engaged in eight different activities that differ widely in their somnificity. These ESS item-scores provide estimates of eight different SSPs for that person [23]. The total ESS score (the sum of 8 item-scores) gives an estimate of a more general characteristic, the person's 'average sleep propensity' or ASP, across a wide range of activities in their daily lives (Johns, 2002). There is no other measure of ASP available at present with which to compare ESS scores directly.

In general, ESS scores can be interpreted as follows:

0-5 Lower Normal Daytime Sleepiness

6-10 Higher Normal Daytime Sleepiness

11-12 Mild Excessive Daytime Sleepiness

13-15 Moderate Excessive Daytime Sleepiness

16-24 Severe Excessive Daytime Sleepiness.

Sleep Hygiene Index [24].

The Sleep Hygiene Index was developed to assess the practice of sleep hygiene behaviors. The Sleep Hygiene Index was delivered to 632 subjects and a subset of the subjects participated in a readministration of the instrument. Test-retest reliability analyses suggested that sleep hygiene behaviors are relatively stable over time for a nonclinical population. Results confirmed that sleep hygiene is strongly related to sleep quality and modestly related to perceptions of daytime sleepiness. As predicted, support of the sleep hygiene construct was also provided by strong correlations with the associated features of a diagnosis of inadequate sleep hygiene. The Sleep Hygiene Index, a much shorter sleep hygiene instrument than previously published, demonstrated comparable psychometric properties with additional evidence of validity and a clear item selection rationale. 
Substance use

The respondents were asked to choose none or more out of tea, coffee, alcohol, cannabis, recreational drugs, prescription medications for an arbitrary question specifically asked as "intake on a regular basis (>3 times/ week)"

\section{Statistical method}

Data were analyzed using the SAS statistical analysis software (version 9.4). The numeric data will be summarized by descriptive statistics like; $\mathrm{n}$, Mean $\pm \mathrm{SD}$, median, minimum, maximum (e.g. Age, BMI, ESS and SHI). The categorical data will be summarized by frequency count and percentage (e.g. Gender, presence of symptom and presence of cause etc.) To find out the statistical significance of numeric data, two sample t-test was used compare SWD and NO SWD group with respect to their characteristics. Significance of categorical data was analyzed using chi-square test e.g. Intake on a regular (Caffeine and Caffeine) basis between SWD and NO SWD group. Logistic regression was used to study statistically significant differences between SWD and their dependent variable. All variables were first entered separately were the $95 \%$ confidence interval, the odds ratios was calculated. The p-value less than 0.05 was considered statistically significant.

Ethical consideration

All forms are anonymous. No identifying information is collected. We will ensure that no clue appears in the study data or analysis, regarding identity of the participant. Volunteer participation will be kept entirely confidential and privacy of the data will be maintained.

Participant data will be available only to the following people or agencies: the Study Doctor and staff; and authorized representatives of the Study Doctor; ethics committees, health authority inspectors, such as (but not limited to) the Drug Controller General of India, designated study monitors and auditors.

\section{Study was initiated only post ISEB approval}

Rights of the participants will not be violated and sufficient measures are described in the protocol for protecting confidentiality of data and privacy of research participant. We request waiver for informed consent form to be obtained from the participants as participation is voluntary.

\section{Results \& Discussion}

A database of approximately 350 email addresses was used. Response rates were $62 \%$. A final sample of 216 was included in analyses. Findings are summarized as Follows

\section{Study Population Characteristics}

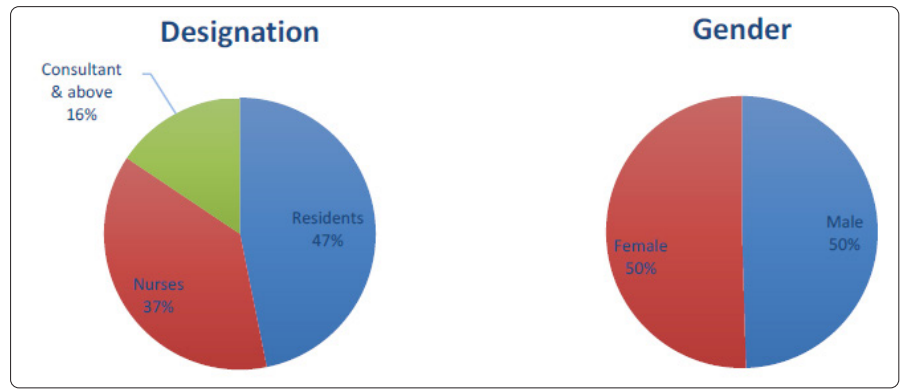

\begin{tabular}{|l|c|c|}
\hline Variable & Mean & Standard deviation \\
\hline Age & 31.76 & 7.72 \\
\hline BMI & 26.82 & 5.89 \\
\hline EM experience (years) & 4.07 & 3.85 \\
\hline Shift work experience (yrs) & 3.28 & 1.65 \\
\hline Avg. night shifts per week & 2.48 & 1.43 \\
\hline Job satisfaction score (1-5) & 3.33 & 0.91 \\
\hline Total ESS & 8.12 & 4.83 \\
\hline Total SHI & 20.86 & 7.98 \\
\hline
\end{tabular}

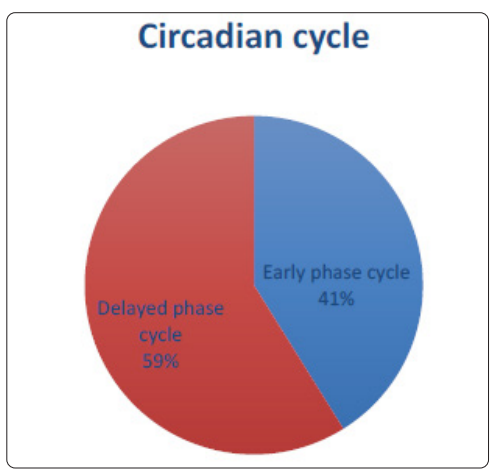

Based on individual self reported preference for the time of day to get important work done - Participants were classified as having an early (morning type person) or delayed (Evening type) phase cycle. Some studies in occupational medicine suggest that people with early phase circadian cycle have more pronounced difficulty in adapting to shift work and sleep schedule irregularities.

In our study, majority participants reported preference for anytime of the day other than early morning indicating that these individuals may be more suited for shifts that start later in the day.

Substance use data is summarized below

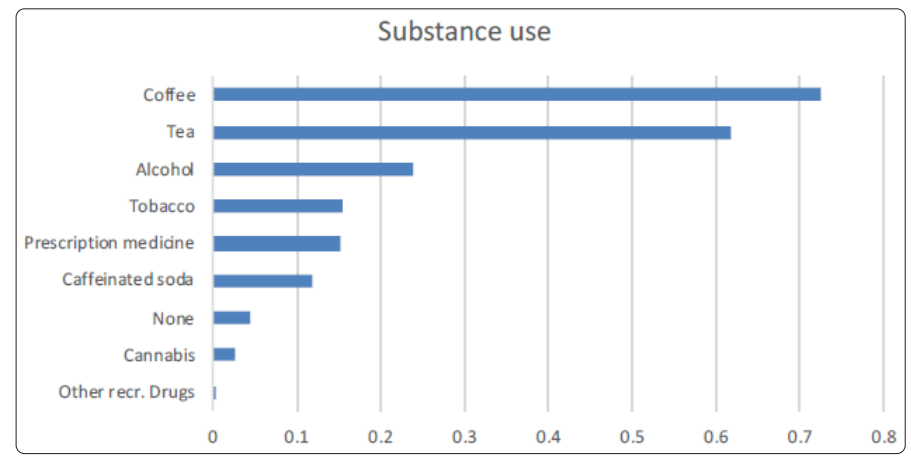

Health, social and occupational problems prevailed as 


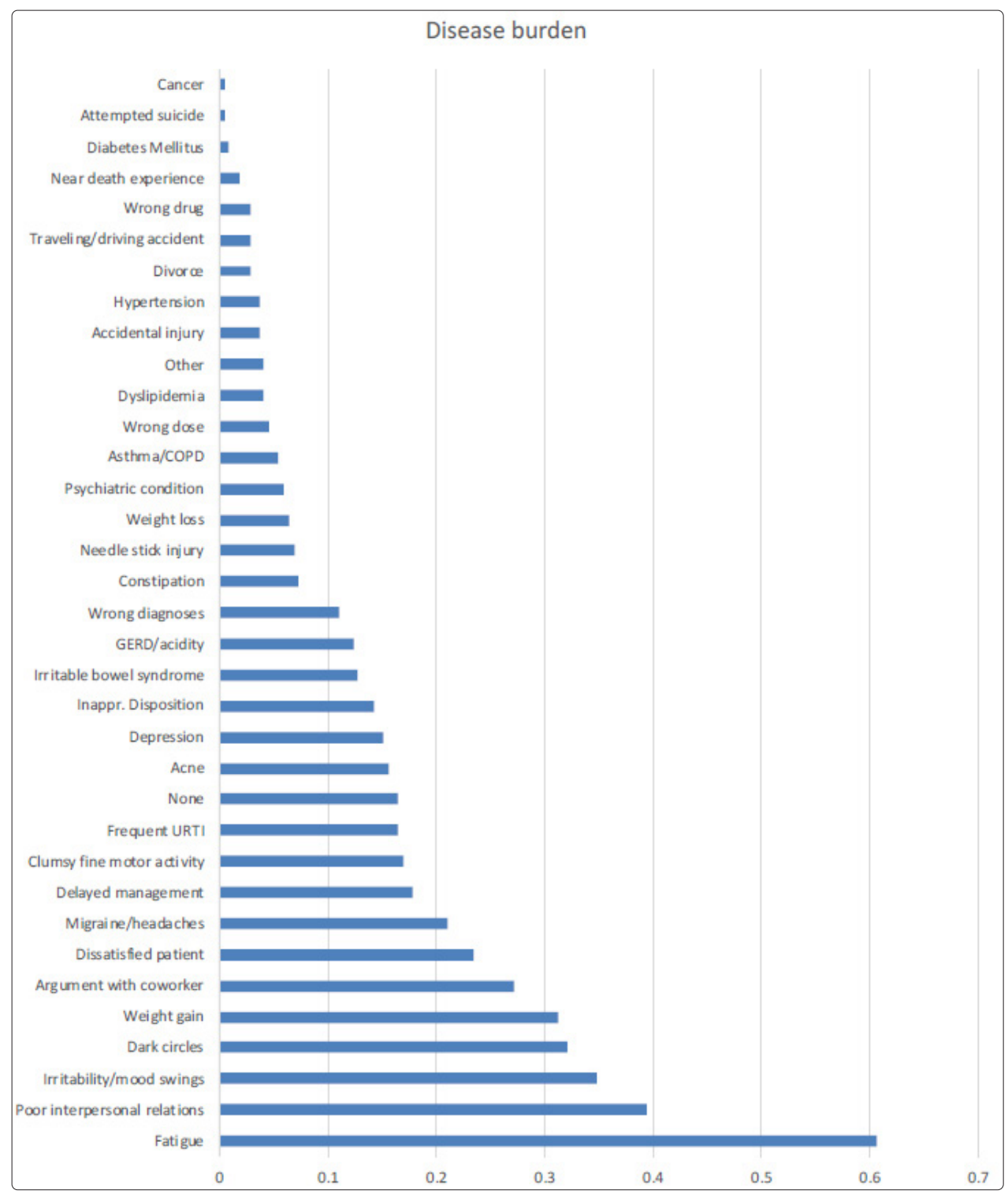

It is not possible to determine, from the current analyses, whether there is any cause-effect relationship between above symptoms and shift work disorder. We also compared people who had above symptoms/diseases to those who had no such complaints. Findings are illustrated in table below. None the less, the prevalence of these symptoms cannot be ignored and adequate measures for promoting wellbeing should be undertaken, starting with strategies for shift work.

\section{Sleep Statistics}

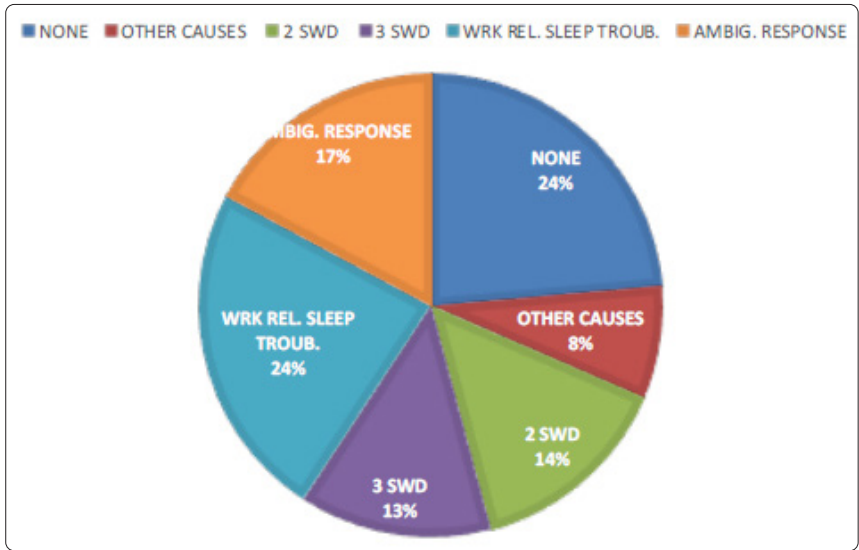


Out of the total sample of 216 participants, approximately $51 \%$ of the people strongly agree to have insomnia/excessive sleepiness pertaining to long working hours or rotational shifts. Out of this, $27 \%$ report that these symptoms cause significant distress in many areas of life. Out of this, close to $13 \%$ report that symptoms have been present for 3 months due to shift work. Thus the estimated prevalence of SWD in our study is $13-27 \%$ based on the method of assessment.

$51 \%$ participants who report insomnia or excessive sleepiness due to reduced sleep time because of work timings, may not be suffering from SWD, they may be prone to developing SWD in the future, if adequate shift work strategies are not designed and implemented.

$38.5 \%$ report significant distress in mental, physical, social, occupational, education or other important areas of functioning due to various causes, highlighting the need for strategies to promote overall health and wellbeing of Emergency medicine providers.

$8.7 \%$ report sleep disturbances from causes other than shift work. Only $24 \%$ report no sleep related symptoms, suggesting that majority of study population is experiencing sleep related trouble in some form or the other.

\section{Swd Subset Characteristics}

\begin{tabular}{|c|c|c|c|c|c|c|c|c|}
\hline & $\mathbf{N}$ & Label & Mean & SD & & $\mathbf{N}$ & Mean & SD \\
\hline \multirow[t]{7}{*}{ No_SWD } & 168 & Age & 31.29 & 7.64 & SWD & 48 & 33.42 & 7.87 \\
\hline & & BMI & 26.98 & 5.99 & & & 26.27 & 5.56 \\
\hline & & Avg. night shifts per week & 2.48 & 1.51 & & & 2.5 & 1.17 \\
\hline & & Total_ESS & 7.67 & 4.62 & & & 9.69 & 5.26 \\
\hline & & Total_SHI & 19.35 & 7.72 & & & 26.15 & 6.55 \\
\hline & & Job satisfaction & 3.4 & 0.94 & & & 3.09 & 0.8 \\
\hline & & Avg. weekly work hrs. & 52.38 & 12.43 & & & 51.46 & 13.53 \\
\hline
\end{tabular}

Above table compares the means and standard deviations between 2 groups -People with symptoms of SWD and those without symptoms of SWD.

\begin{tabular}{|l|c|}
\hline Label & P value \\
\hline Gender & 0.8635 \\
\hline Age & 0.071 \\
\hline BMI & 0.5291 \\
\hline Caffeine & 0.3286 \\
\hline Alcohol & 0.7662 \\
\hline Average hours do you work in a week & 0.3594 \\
\hline Shift_Work_Yes_or_No & 0.0485 \\
\hline Avg. weekly night shifts & 0.7341 \\
\hline Night Shift Work (Yes or No) & 0.0485 \\
\hline Total_ESS & 0.0158 \\
\hline Total_SHI & $<.0001$ \\
\hline Overall personal Job satisfaction & 0.037 \\
\hline How long have you been doing Shift work? & 0.7325 \\
\hline Designation & 0.8696 \\
\hline Sleeping aids & 0.0014 \\
\hline Morning preference & 0.51 \\
\hline
\end{tabular}

On further analyses, statistically significant difference was found between the 2 groups in terms of doing shift work, night shift work, mean total ESS, mean total SHI, mean job satisfaction scores and the use of sleeping aids. We compared the same parameters in those who reported sleep disturbances from other causes and findings are summarized below.

In our study no statistical correlation was found between SWD and gender, caffeine and alcohol consumption, number of night shifts, average weekly working hours. Though those who reported caffeine consumption also reported significantly low ESS scores suggesting, caffeine may be effective at promoting alertness. 


\begin{tabular}{|c|c|c|c|c|c|c|c|}
\hline \multicolumn{8}{|c|}{ Odds Ratio Table } \\
\hline \multirow[t]{2}{*}{ Parameter } & \multirow[t]{2}{*}{ Estimate } & \multirow[t]{2}{*}{ SE } & \multirow[t]{2}{*}{ Wald } & \multirow[t]{2}{*}{ p } & \multicolumn{3}{|l|}{ Odds Ratio } \\
\hline & & & & & Point Estimate & 95\% Walc & ce Limits \\
\hline C (Age_Range) & -0.1212 & 0.1808 & 0.4493 & 0.5027 & 0.886 & 0.622 & 1.263 \\
\hline $\mathrm{D}$ (Gender) & 1.1007 & 0.5401 & 4.1524 & 0.0416 & 3.006 & 1.043 & 8.665 \\
\hline $\begin{array}{l}\mathrm{H}(\text { Intake on a regular basis } \\
(>\text { once a week) })\end{array}$ & 0.0042 & 0.0230 & 0.0334 & 0.8550 & 1.004 & 0.96 & 1.051 \\
\hline $\begin{array}{l}\text { I (How many hours do you work in a } \\
\text { week on average?) }\end{array}$ & 0.0122 & 0.1871 & 0.0042 & 0.9482 & 1.012 & 0.702 & 1.461 \\
\hline $\mathrm{J}$ (My work schedule is) & -0.0794 & 0.0513 & 2.4023 & 0.1212 & 0.924 & 0.835 & 1.021 \\
\hline $\begin{array}{l}\mathrm{K} \text { (How many night shifts do you } \\
\text { work in a week on average?) }\end{array}$ & 0.2582 & 0.1455 & 3.1509 & 0.0759 & 1.295 & 0.973 & 1.722 \\
\hline $\begin{array}{l}\text { NN (Years of Emergency medicine } \\
\text { experience) }\end{array}$ & 0.0293 & 0.0689 & 0.1815 & 0.6701 & 1.03 & 0.9 & 1.179 \\
\hline $\begin{array}{l}\text { OO (When do you prefer to get } \\
\text { important work done?) }\end{array}$ & 0.5368 & 0.4825 & 1.2377 & 0.2659 & 0.705 & 0.54 & 0.92 \\
\hline PP (Sleeping aids) & 0.1752 & 0.0840 & 4.3528 & 0.0369 & 1.191 & 1.011 & 1.405 \\
\hline QQ (Overall personal Job satisfaction) & 0.1214 & 0.2689 & 0.2039 & 0.6516 & 1.129 & 0.667 & 1.913 \\
\hline $\begin{array}{l}\text { RR (How long have you been doing } \\
\text { Shift work?) }\end{array}$ & 0.1009 & 0.1410 & 0.5118 & 0.4743 & 1.106 & 0.839 & 1.458 \\
\hline SS (Designation) & 0.0474 & 0.1667 & 0.0808 & 0.7762 & 1.049 & 0.756 & 1.454 \\
\hline TT (BMI Range) & 0.0199 & 0.2045 & 0.0095 & 0.9225 & 1.02 & 0.683 & 1.523 \\
\hline
\end{tabular}

The odds of a woman having SWD are thrice the odds of a man.

Advantages and Disadvantages of Web-Based Questionnaire Study (25)

Advantages

Data collection using Web-based questionnaires generally improves data quality since validation checks can be incorporated with prompts that alert respondents when they enter implausible or incomplete answers. Even without forced-choice formats, item nonresponse and "don't know" answers are reported to be less prevalent in Web-based questionnaires compared with postal questionnaires.

Because data are entered electronically and may automatically be transformed into an analyzable format by common gateway interface (CGI) scripts, errors in the process of data entry and coding are avoided as well. Common gateway interface scripts can also be used to build in skip patterns to hide non-relevant follow-up questions, order questions randomly, give personalized feedback, or randomize participants to different versions of the questionnaire. Visual and audio aids and pop-up windows providing additional information may be added to simplify responding, which is impossible in paperand-pencil questionnaires. However, all these additional features will increase download time, which may contribute to nonresponse.

Experience shows that Web-based questionnaires are returned more rapidly than postal questionnaires, with most respondents completing the questionnaire within a few days. Completing all questions in a Web-based questionnaire was estimated to take about half the time needed to answer the same number of questions in a telephone interview. Researchers are able to immediately adjust Web-based questionnaires to resolve unforeseen problems or to incorporate preliminary results or new developments. A data management system may be used to automatically send e-mail reminders and invitations for follow-up questionnaires to study participants, although followup of "bounce-back" (undeliverable) e-mails will be time-consuming.

Although some authors state that the use of Web-based questionnaires results in substantial cost reductions, others conclude that the cost savings are currently unknown. With Web-based questionnaires, costs for printing, postage, and data entry are avoided, but the setup costs, including Web site and survey design, may be substantial, although the marginal costs for adding more participants to the study are relatively low. Therefore, the cost per response may be high when Web-based questionnaires are used in studies with small sample sizes or in populations with low response rates to Web surveys. Studies that invited participants through e-mail reported cost benefits associated with using Web-based questionnaires.

\section{Disadvantages}

Two main disadvantages that may hamper the use of Web-based questionnaires in epidemiological research were identified at the beginning of this century: 1) relatively high nonresponse rates compared with traditional modes of data collection and 2) concerns regarding the reliability and validity of the data obtained. 3) Poor internet access in India leading to poor response rates.

\section{Conclusions}

a. Prevalence rate of symptoms of SWD in emergency medicine providers in India is $13-27 \%$. Symptoms of SWD occurred more frequently in people who performed shift work and night shift work compared to those who did to work in shifts. $(p=0.048)$.

b. Women are 3 times more likely to have SWD than their male counterparts. $(p=0.0416)(O R=3.006,95 \% C I-1.043-8.665)$

c. Shift work disorder is strongly associated with bad sleep hygiene. $(\mathrm{p}<0.0001$ for SHI score $>26)$ Whether shift work leads to bad sleep hygiene practices that results in shift work disorder is subject to further research.

d. Though SWD is associated with higher ESS $\operatorname{scores}(p=0.015)$, 
SWD patients may have normal ESS scores. Having a normal ESS score does not exclude SWD or other sleeping disorders.

e. People with SWD reported lower job satisfaction scores $(p=0.037)$.Despite all the health and sleep problems, $(66.5 \%)$ majority of the study population report high job satisfaction $\operatorname{scores}(>3 / 5)$, reasons for which are subject to further research.

f. People with SWD are more likely to use sleeping aids. $(\mathrm{p}=0.0014)(\mathrm{OR}=1.19,95 \% \mathrm{CI}-1.011-1.405$. $)$

g. Majority $(60 \%)$ of study population can be said to have a delayed phase sleep cycle(evening type person), thus making them likely to function or perform better in shifts that start later in the day. Whereas the other $40 \%$ with early phase cycle(morning type person) may be more suitable for early morning shifts. Even though there is no statistical data to support this, individual should ideally work in whichever shift is best preferable to $\operatorname{him} /$ her.

h. Mean age of emergency medicine providers in India is approximately 31 years underlining the fact that it is a young and upcoming specialty.

i. Mean BMI of study population is 26, suggesting most of our study population borders on overweight category.

j. Commonest $(>15 \%)$ health complaints recorded were fatigue, poor interpersonal relationships, irritability/mood swings, dark circles, weight gain, migraine, depression, irritable bowel, GERD, frequent URTIs among many others.

k. Commonest occupational complaints were argument with coworker, dissatisfied patient, delayed management, inappropriate disposition and clumsiness in fine motor activity.

\section{References}

1. Chapman DM (1997) Burnout in emergency medicine: What are we doing to ourselves?. Acad Emerg Med 4: 245-247.

2. Zun L, Kobernick M, Howes DS (1988) Emergency physician stress and morbidity. Am J Emerg Med 6: 370-374.

3. Maslach C, Jackson SE (1981) Maslach Burnout Inventory. Palo Alto, Calif: Consulting Psychologists Press.

4. Maslach C, Jackson SE (1986) The Maslach Burnout Inventory, $2^{\text {nd }}$ ed. Palo Alto, Calif: Consulting Psychologists Press.

5. Maslach C, Jackson SE (1984) Patterns of burnout among a national sample of public contact workers. J Health Hum Res Admin 76: 189-212.

6. Centrella M (1994) Physician addiction and impairment-current thinking: A review. J Addict Dis 13: 91-105.

7. Quill TE, Williamson PR (1990) Healthy approaches to physician stress. Arch Intern Med 1990; 150: 1857-1861.

8. Covey S (1989) The Seven Habits of Highly Effective People: Restoring the Character Ethic. New Y ork: Simon \& Shuster.
9. Margulies JL, Pollack ML (1995) Developing a healthy lifestyle. In: Andrew LB, Pollack ML, eds: Wellness for Emergency Physicians. Dallas, Tex: American College of Emergency Physicians; 1995: 24-26.

10. American Academy of Sleep Medicine (2014) International Classification of Sleep Disorders, $3^{\text {rd }}$ ed, American Academy of Sleep Medicine, Darien, IL 2014.

11. Keller KL, Koenig WJ (1989) Sources of stress and satisfaction in emergency practice. J Emerg Med 7: 293-299.

12. Lloyd S, Streiner D, Shannon S (1994) Burnout, depression, life and job satisfaction among Canadian emergency physicians. J Emerg Med 12: 559-265.

13. Margulies JL (1995) Control issues. In: Andrew LB, Pollack ML, eds: Wellness for Emergency Physicians. Dallas, Tex: American College of Physicians.

14. Dula DJ, Dula NL, Hamrick C, Wood GC (2001) The effect of working serial night shifts on the cognitive functioning of emergency physicians. Ann Emerg Med 38: 152-155.

15. Kuhn G (2001) Circadian rhythm, shift work, and emergency medicine. Ann Emerg Med 37: 88-98.

16. Silbergleit R (1982) Shift work and physician performance. Ann Emerg Med 39: 349-351.

17. Culpepper L (2010) The social and economic burden of shiftwork disorder. J Fam Pract 59: 3-11.

18. Costa G (1986) The impact of shift and night work on health. Appl Ergon 27: 9-16.

19. Park YM, Matsumoto PK, Seo YJ, Cho YR, Noh TJ, et al. (2000) Sleep-wake behavior of shift workers using wrist actigraph. Psychiatry Clin Neurosci 54: 359-360.

20. Pilcher JJ, Lambert BJ, Huffcutt AI (2000) Differential effects of permanent and rotating shifts on self-report sleep length: a meta-analytic review. Sleep 23: 155-163.

21. Killgore WD (2010) Effects of sleep deprivation on cognition. Prog Brain Res. 185: 105-129.

22. DI Milla L, Waage S, Pallesen S, Bjorvatn B (2013) Shift Work Disorder in a Random Population Sample - Prevalence and Comorbidities. PLoS ONE 8: e55306.

23. Johns M W (2000) Sensitivity and specificity of the multiple sleep latency test (MSLT), the maintenance of wakefulness test and the Epworth sleepiness scale: Failure of the MSLT as a gold standard. Journal of Sleep Research 9: 5-11.

24. Mastin DF, Bryson J, Corwyn R, J Behav Med (2006) Assessment of sleep hygiene using the Sleep Hygiene Index 29: 223-227.

25. Web-based Questionnaires (2010) The Future in Epidemiology? Marleen M. H. J. van Gelder, Reini W. Bretveld, Nel Roeleveld Am J Epidemiol 172: 1292-1298.

Citation: Dharmik Vora, Sanjay Mehta, Shashank Patil (2019) Shift Work Disorder - Prevalence \& Burden of Illness in Healthcare Providers of India. Med Clin Res 4(6): 1-9.

Copyright: (C2019 Dharmik Vora, et al. This is an open-access article distributed under the terms of the Creative Commons Attribution License, which permits unrestricted use, distribution, and reproduction in any medium, provided the original author and source are credited. 